\title{
Tulane
}

\author{
Tulane Economics Working Paper Series
}

\section{Optimal Provision of a Discrete Public Good: Linear Equilibria in the Private-Information Subscription Game}

\author{
Stefano Barbieri \\ Department of Economics \\ 206 Tilton Hall \\ Tulane University \\ New Orleans, LA 70118 \\ sbarbier@tulane.edu
}

\author{
David A. Malueg \\ Department of Economics \\ 3136 Sproul Hall \\ UC Riverside \\ Riverside, CA 92521-0427 \\ david.malueg@ucr.edu
}

Working Paper 0902
March 2008

\begin{abstract}
We analyze a symmetric Bayesian game in which two players individually contribute to fund a discrete public good; contributions are refunded if they do not meet a threshold set by the seller of the good. We provide a general characterization of symmetric equilibrium strategies that are continuous and nonconstant over the set of values for which the good has a positive chance of provision. Piecewiselinear strategies are our special focus. We characterize the distributions of players' private values that can support a continuous piecewise-linear symmetric equilibrium, and we calculate such equilibria for these distributions. Allowing the seller to charge a nonrefundable entry fee before players make their private contributions, we show these piecewise-linear equilibria can maximize the seller's expected utility, which may include an altruistic component, over all incentive compatible selling mechanisms.
\end{abstract}

Keywords: discrete public good, subscription game, Revelation Principle JEL codes: H41, D61, D82 


\title{
Optimal Provision of a Discrete Public Good: linear equilibria in the private-information subscription game
}

\author{
Stefano Barbieri* David A. Malueg ${ }^{\dagger}$
}

March 17, 2008

\begin{abstract}
We analyze a symmetric Bayesian game in which two players individually contribute to fund a discrete public good; contributions are refunded if they do not meet a threshold set by the seller of the good. We provide a general characterization of symmetric equilibrium strategies that are continuous and nonconstant over the set of values for which the good has a positive chance of provision. Piecewise-linear strategies are our special focus. We characterize the distributions of players' private values that can support a continuous piecewise-linear symmetric equilibrium, and we calculate such equilibria for these distributions. Allowing the seller to charge a nonrefundable entry fee before players make their private contributions, we show these piecewise-linear equilibria can maximize the seller's expected utility, which may include an altruistic component, over all incentive compatible selling mechanisms.
\end{abstract}

JEL Codes: H41, D61, D82

Keywords: discrete public good, subscription game, Revelation Principle

\section{DRAFT: Comments welcome}

\footnotetext{
*Department of Economics, 206 Tilton Hall, Tulane University, New Orleans, LA 70118; email: sbarbier@tulane.edu.

${ }^{\dagger}$ Department of Economics, 3136 Sproul Hall, University of California, Riverside, CA 92521; email: david.malueg@ucr.edu.
} 


\section{Introduction}

A discrete public good is either provided or not - quantity is not otherwise variable. The subscription game is a voluntary provision mechanism for such goods: Individuals privately contribute money; if contributions suffice to fund the good provision occurs, otherwise contributions are refunded. An example is a homeowners' association collecting pledges to fund a new swimming pool. If enough money is pledged, it will then be collected and the pool built; otherwise, no pool is built and no money is paid.

This game and the closely related contribution game have been widely analyzed in the literature in a variety of different economic environments. ${ }^{1}$ For example, the complete-information case is well-understood, both for static and for dynamic problems (e.g. Palfrey and Rosenthal, 1984; Bagnoli and Lipman, 1989; Admati and Perry, 1991; and Marx and Matthews, 2000). In the more realistic case where valuations for the public good are private information, however, many questions remain unanswered, even restricting attention to static, two-player subscription games (cf. Alboth et al., 2001; Menezes et al., 2001; Laussel and Palfrey, 2003; and Barbieri and Malueg, forthcoming (b)). From a positive point of view, the common message of these private-information papers is that a profusion of equilibria exists and analytical characterizations are very difficult. Therefore, these papers mostly focus on a uniform distribution of agents' valuations. From a normative point of view, the main focus of the previous analyses is efficiency-neither the contribution game nor the subscription game is clearly superior. Menezes et al. (2001) show classical inefficiency of both subscription and contribution games. Laussel and Palfrey (2003) and Barbieri and Malueg (forthcoming (b)) use the less demanding notion of interim incentive efficiency. ${ }^{2}$ Even for valuations uniformly distributed on $[0,1]$, results are mixed. Indeed, Laussel and Palfrey (2003) show interim incentive efficiency of the subscription game when the cost of provision is sufficiently high. In contrast, Barbieri and Malueg (forthcoming (b)) show interim incentive inefficiency of the subscription game equilibria when the cost of provision is sufficiently low. In addition, Barbieri and Malueg (forthcoming (a)) show interim incentive efficiency for the contribution game, albeit only for a uniform distribution of valuations. ${ }^{3}$

Of particular interest to economists is understanding when a simple indirect mechanism such as the subscription game can achieve some theoretical desiderata. Among these desiderata, efficiency occupies an important place, but other objectives remain interesting. Indeed, in this paper we provide a justification for the use of the subscription game, complementary to the efficiency analysis described above, that focuses on

\footnotetext{
${ }^{1}$ The contribution game is identical to the subscription game except that contributions are never refunded.

${ }^{2}$ Classical and interim incentive efficiency are discussed in Holmström and Myerson (1983). Interim efficiency in a public goods economy is analyzed in detail in Ledyard and Palfrey (1999).

${ }^{3}$ In a continuous-public-goods economy, that is with variable quantity, another paper that focuses on interim incentive efficiency is Martimort and Moreira (2007). They analyze a common agency game and show how interim incentive efficiency becomes much harder to achieve in comparison to discrete public goods economies. Despite the differences between the games Martimort and Moreira (2007) and we study, some technical similarities arise. We will analyze them in detail in Section 2.
} 
the incentives of the producer of the public good. As in the papers mentioned above, we consider a static, two-player subscription game. A central role in our analysis is played by an additional agent: the producer (variously referred to as organizer, seller or collector). This agent specifies a contribution threshold, provides the public good, and retains contributions in excess of the threshold. ${ }^{4}$ We specify an objective for the collector that is a mix of profit-maximization and altruism towards the players. We then ask the following natural question: Would the organizer ever choose the subscription game as an optimal provision mechanism, among all incentive compatible ones? We show the answer is Yes. We then ask the same question about the contribution game and show, generically, the answer is No.

Our results do not require the collector's participation in the financing of the public good when the collector is a pure profit-maximizer. In this case, our main contribution bridges two strands of the literature on the profit-maximizing behavior of producers of public goods. On the one hand, Cornelli (1996) characterizes the profit-maximizing direct mechanism for the sale of a pure public good. On the other hand, Alboth et al. (2001) consider the problem of a profit-maximizing entrepreneur who has already chosen the subscription game as a selling mechanism. Our analysis considerably extends the results in Alboth et al. (2001). In a narrow sense, we relax various distributional and parametric assumptions in that paper. More importantly, we exhibit environments in which no other selling mechanism outperforms the subscription game.

Beyond profit maximization, we allow the seller to exhibit a degree of altruism, in which case the seller's profit, though not utility, can be negative. Therefore, our positive result for the subscription game does not conflict with the classical inefficiency results in Myerson and Satterthwaite (1983) and Menezes et al. (2001), because we allow the collector to inject resources to "grease the wheels" of the public good provision problem. Indeed, when the altruistic motivation is sufficiently important, the collector optimally chooses a contribution threshold lower than the full cost of provision. Such strategy may be interpreted as a "matching" grant: As long as players' contributions reach a certain threshold, the collector commits to filling in any remaining financing needs to bring the project to completion. ${ }^{5}$ In other words, when the altruistic motivation is important, our collector is interested in helping players overcome the free-rider problem, but, at the same time, it is trading off helping players with devoting its resources towards other uses, whose marginal utility is normalized to unity. Our optimality result shows that, through the implicit matching grant just described, the subscription game may allow the collector to share in the financing of the public good only when players' valuations are sufficiently large, and, at the same time, to minimize its financial outlay.

A necessary preliminary step in our analysis is to sharpen the existing equilibrium characterization results.

\footnotetext{
${ }^{4}$ The possibility of choosing a threshold different than the cost of production distinguishes our collector from that in Laussel and Palfrey (2003) and Martimort and Moreira (2007). Unlike in Alboth et al. (2001), our collector may choose a contribution threshold lower than the cost of provision, therefore effectively participating in the financing of the public good (in Alboth et al. the cost of provision is zero).

${ }^{5}$ Examples of matching grants abound in the literature; for one example among many see Andreoni (1998).
} 
As in literature previously mentioned, we study a two-player subscription game in which players' values for the public good are private information, characterized by a continuous distribution function. We provide a general characterization of symmetric equilibrium strategies that are continuous and nonconstant over the set of values for which the good has a positive chance of provision. Our analysis completes the results in Menezes et al. (2001) and Laussel and Palfrey (2003), and it connects and contrasts them with those in Martimort and Moreira (2007). Beyond our general characterization, we are particularly interested in equilibrium strategies that are piecewise-linear and are also strictly increasing over some interval of possible values. We show that for linear equilibria to exist, players' values must be distributed according to an exponential distribution that does not include zero in its support or a reverse power function distribution (of which the uniform distribution is a special case). ${ }^{6}$ Cornelli (1996) adapted Myerson's (1981) method of optimal auction design to characterize an optimal incentive compatible mechanism for the sale of a discrete public good. Within our framework, we show that, for the exponential and reverse power function distributions, the piecewise-linear equilibria of the subscription game (possibly with the introduction of nonrefundable entry fees) achieve the expected payoff of an optimal mechanism for the seller; and if such piecewise-linear equilibria do not exist, then the subscription game cannot be an optimal mechanism for the seller. When piecewise-linear equilibria exist, we completely describe the seller's optimal choice of completion threshold and entry fee. We conclude by establishing a generic suboptimality result for the contribution game.

The rest of the paper is organized as follows. Section 2 defines "regular" equilibria and provides a general characterization result. In Section 3 we completely characterize piecewise-linear equilibria. Section 4 contains our main result on the optimality of the subscription game and relates it to the existing literature and to the contribution game. Section 5 concludes.

\section{The subscription game}

Two players, 1 and 2, simultaneously contribute any positive amount to the funding of a public good. Player $i$ 's value for the good is $v_{i}, i=1,2$. Values $v_{1}$ and $v_{2}$ are independently distributed random variables with cumulative distribution function (cdf) $F$, which has support $[\underline{v}, \bar{v}]$, where $0 \leq \underline{v}<\bar{v} \leq \infty$. A player's realized value is known only to that player. We suppose $F$ is absolutely continuous, with density function $f$. The third actor in the model is the collector, who ex ante specifies a contribution threshold $t$ and provides the public good if and only if contributions total at least $t$. The cost of the public good is $c$, known to the collector and both players.

\footnotetext{
${ }^{6}$ There are other distributions that also support such piecewise-linear equilibrium strategies, but they would differ from the two families named only over types where (i) a player has no chance of obtaining the good or (ii) a player is sure he will obtain the good. These alternative distributions must agree with the families named over the interval of values where the piecewise-linear strategy is strictly increasing.
} 
In the terminology of Admati and Perry (1991), we consider the subscription game: players' contributions are refunded if they are insufficient to cover $t$; the collector retains contributions exceeding $t$. The collector and two players are risk neutral. If the good is provided, then the payoff to player $i$ is $v_{i}-$ (player $i$ 's contribution). If the good is not provided, then the payoff to player $i$ is 0 .

The foregoing description is common knowledge among the players and the collector. We focus on symmetric equilibrium strategies $(s, s)$ such that completion happens with ex ante positive probability. In this case, standard arguments show $t<2 \bar{v}$ and $s$ is nondecreasing over all types having a positive probability of provision.

At this point some additional notation is useful. Define $\hat{v}$ as the lowest type who contributes the maximum under $s$, and define $\widetilde{v}$ as the lowest type who contributes $t-s(\hat{v})$. For simplicity, our characterization is for strategies $s$ that achieve their maxima at finite $\hat{v}^{7}$ Thus,

$$
\begin{array}{ll}
\hat{v} \equiv \inf \left\{v \mid s(v)=\max _{v} s(v)\right\} & \text { (lowest type contributing the maximum) } \\
\widetilde{v} \equiv \inf \{v \mid s(v) \geq t-s(\hat{v})\} . & \text { (lowest type with positive probability of provision of the good) }
\end{array}
$$

Because the interim probability of completion for type $v \in[\underline{v}, \widetilde{v})$ is zero, little more can be said about the shape of $s$ on $[\underline{v}, \widetilde{v})$. To avoid uninteresting complications, we set $s=0$ in this range. Lemmas 1-3 in Laussel and Palfrey (2003) establish that on $[\widetilde{v}, \bar{v}]$ a symmetric equilibrium contribution function $s$ is non-decreasing and almost everywhere differentiable. A first possibility is that $s$ is a step function. If an appropriate boundary condition, later described in (4), is satisfied, then the following strategy constitutes the "halvesies" equilibrium: $s(v)=0$ for $v \in[\underline{v}, t / 2)$ and $s(v)=t / 2$ for $v \in[t / 2, \bar{v}]$. Menezes et al. (2001) point out, for $t<\bar{v}$, the "all-or-nothing" equilibrium is sure to exist: $s(v)=0$ for $v<v^{*}$ and $s(v)=t$ for $v \geq v^{*}$, where $v^{*}$ solves $v F(v)=t$. As will be seen later, however, if the subscription game is to be an incentive compatible mechanism that is optimal for the seller, then strategies must be continuous and strictly increasing in the region where a player's chance of obtaining the good is strictly between 0 and 1. These considerations lead us to focus on "regular" equilibria.

Definition 1. A symmetric equilibrium $(s, s)$ is called regular if $s$ is continuous and nonconstant on $[\widetilde{v}, \bar{v}]$.

Note that neither the halvesies equilibrium nor the all-or-nothing equilibrium is regular. Barbieri and Malueg (forthcoming (b)) establish that in a regular equilibrium $s$ must be strictly increasing on $[\widetilde{v}, \hat{v}]$; furthermore $\widetilde{v}$ and $\hat{v}$ are not at all arbitrary, but rather are jointly determined as part of the equilibrium. We now characterize regular equilibria.

\footnotetext{
${ }^{7}$ The characterization can be extended to cases where $\hat{v}=\infty$ by replacing $s(\hat{v})$ with the appropriate limiting value of $s$.
} 
Proposition 1 (Characterization of a regular symmetric equilibrium). Suppose $s:[\underline{v}, \bar{v}] \rightarrow \mathbf{R}_{+}$has the following properties, where $\widetilde{v}$ and $\hat{v}$ are defined above:

a. $\underline{v} \leq \widetilde{v}<\hat{v} \leq \bar{v}$

b. $s(v)=0$ for any $v<\widetilde{v} ; s$ is continuous and nondecreasing on $[\widetilde{v}, \bar{v}]$;

c. $s(\widetilde{v})+s(\hat{v})=t ;$ and

d. $s$ is strictly increasing on $(\widetilde{v}, \hat{v})$.

Define $G:[\widetilde{v}, \hat{v}] \rightarrow[\widetilde{v}, \hat{v}]$ by $s(v)+s(G(v))=t$. Then $(s, s)$ is a regular symmetric equilibrium to the subscription game with threshold $t$ if and only if $s$ and $G$ are differentiable on $(\widetilde{v}, \hat{v})$ and satisfy the following system of equations:

$$
\begin{array}{r}
s^{\prime}(v)(1-F(G(v)))+(v-s(v)) f(G(v)) G^{\prime}(v)=0 \quad \forall v \in(\widetilde{v}, \hat{v}), \\
s^{\prime}(v)+s^{\prime}(G(v)) G^{\prime}(v)=0 \quad \forall v \in(\widetilde{v}, \hat{v}), \\
0 \leq s(v) \leq v \quad \forall v \in(\widetilde{v}, \bar{v}) ; \\
\text { and further, if } \widetilde{v}>\underline{v} \text {, then } s(\widetilde{v})=\widetilde{v} \text { and }(v-s(v))(1-F(G(v))) \geq v-t \quad \forall v \geq \hat{v} .
\end{array}
$$

Conditions $a-d$ describe a candidate strategy for a regular symmetric equilibrium. Condition $a$ rules out the possibility that $s$ is constant on $[\widetilde{v}, \bar{v}$ ( the halvesies equilibrium satisfies $b-d$ because $\widetilde{v}=\hat{v}=t / 2$ so the interval $(\widetilde{v}, \hat{v})$ is empty). Since $s$ is strictly increasing on $(\widetilde{v}, \hat{v})$, the definition of $G$ implies that, in equilibrium, type $v \in(\widetilde{v}, \hat{v})$ will see the threshold for completion reached if and only if the other player's type is at least as large as $G(v)$.

Menezes et al. (2001) showed that a continuous equilibrium to the subscription game must satisfy (1) and (2). The value of Proposition 1 is that, with the addition of the boundary conditions (3) and (4), it completes their equilibrium characterization. Our proposition also shows that continuity of the equilibrium strategy over $[\widetilde{v}, \bar{v}]$ implies differentiability over $[\widetilde{v}, \hat{v}]$. The reader may be curious why the inequality in (4) applies only in the case where $\widetilde{v}>\underline{v}$. This condition says that a player contributing the maximum, $s(\hat{v})$, should not strictly benefit by contributing $t$ (thereby ensuring the good is provided). This condition is not included when $\widetilde{v}=\underline{v}$ because in that case it is automatically satisfied. To see this, observe that if $\widetilde{v}=\underline{v}$, then $s(\underline{v})+s(\hat{v})=t$, so that a player contributing $s(\hat{v})$ is assured the good will be provided-hence, there is no profitable deviation to the (possibly) larger contribution $t$.

In their Proposition 1, Laussel and Palfrey (2003), with a different approach, provide conditions characterizing equilibrium equivalent to our conditions (1)-(3). The only substantive difference between our 
characterization and Laussel and Palfrey's is that we further impose condition (4). ${ }^{8}$ We believe our condition (4) clarifies and sharpens the characterization of equilibria. To see this, consider valuations distributed on $[0,1]$ with density $f(v)=2(1-v)$ and the halvesies strategy described above. Apparently, all conditions in Laussel and Palfrey's Proposition 1 are satisfied. However, one may verify that type $\bar{v}$ prefers to deviate to the full contribution of $t$ as soon as $t<3-\sqrt{5}$. Equivalently, our condition (4) is not satisfied for $t<3-\sqrt{5}$. This is because the local first-order condition (1), obtained through the revelation principle, does not eliminate contributions outside the range of $s$ as more profitable. Our condition (4) provides a simple test for global optimality of $s$. Indeed, to the local first-order condition (1), one simply needs to add the constraint that completing the project on one's own is not profitable. Moreover, it is worth noting that, when $\bar{v}<\infty$, condition (4) will hold for any $v \geq \hat{v}$ if and only if it holds for $\bar{v}$, thereby providing a further sharpening of the equilibrium characterization.

Martimort and Moreira (2007) point out the similarity between the equilibrium characterization of their common agency game and the results in Menezes et al. (2001); therefore the similarity extends to our Proposition 1. Martimort and Moreira are interested in equilibria that, in our framework, are regular and with $0<s^{\prime}(v)<\infty$. They show existence of such equilibria, with $\hat{v}=\bar{v}$, under the additional assumption that $\left(\frac{1-F(v)}{f(v)}\right)^{\prime} \leq-1$, or equivalently, that $f^{\prime}(v) \geq 0$. In this case, one can show that our condition (4) is automatically satisfied for such regular equilibria. However, there exist many regular equilibria that do not satisfy the condition $0<s^{\prime}(v)<\infty$ at $v=\bar{v}$, as Figure 1 in Alboth et al. (2001) shows.

Regular equilibria with a flat spot at the top, that is with $\hat{v}<\bar{v}$, also play an important role in our optimality results. Indeed, in Section 3 we exhibit various examples of regular equilibria $(s, s)$ with $\hat{v}<\bar{v}$ and $s(\hat{v})<t$. It is worth noting that such equilibria are not considered in any of the papers in the literature on the subscription game, with the exception of Barbieri and Malueg (forthcoming (b)) for the specific case of a uniform distribution of valuations. ${ }^{9}$ The characterization in Proposition 1 can be used to refine our understanding of regular equilibria, especially of those exhibiting a flat spot at the top. A straightforward but tedious application of the contraction mapping theorem demonstrates that, if $\underline{v}>0$ and $f \in C^{1}$, a regular equilibrium with a flat spot at the top is sure to exist for $t$ sufficiently small. More interesting is the following proposition showing that $\hat{v}<\bar{v}$ is incompatible with $\widetilde{v}>\underline{v}$ because the latter implies, by condition (4), that $s(\widetilde{v})=\widetilde{v}$. This result considerably sharpens the equilibrium characterization: One of the extremes of the interval $[\widetilde{v}, \hat{v}]$ where Proposition 1 must hold is always fixed.

Proposition 2 (Necessary condition for existence of flat spots). Suppose on $[\underline{v}, \bar{v})$ the density $f$ is continuous

\footnotetext{
${ }^{8}$ To go from Laussel and Palfrey's formulation to our Proposition 1 it is enough to differentiate their conditions (2.3) and (2.5) on page 455. The only difference in notation is that our function $G$ corresponds to their function $\Phi$. Note as well how Laussel and Palfrey's result holds for all equilibria, not just regular ones.

${ }^{9}$ Laussel and Palfrey's semi-regular equilibria do have a flat spot at the top for $s(\hat{v})=t$ and a flat spot at the bottom. Barbieri and Malueg (forthcoming (b)) show this class of equilibria is empty.
} 
and strictly positive. If $(s, s)$ is a regular equilibrium with $\hat{v}<\bar{v}$, then $s(\widetilde{v})<\widetilde{v}$.

Proposition 2 has important consequences for the welfare properties of the subscription game. For the particular case in which values are uniformly distributed over $[0, \bar{v}]$, Barbieri and Malueg (forthcoming (b)) show that all equilibria are interim incentive inefficient if $c<\bar{v}$. Proposition 2 extends this negative result to all continuous distributions on $[0, \bar{v}]$ that are strictly positive at $\underline{v}=0$. The reason for this is that an incentive efficient equilibrium, for reasons discussed in Barbieri and Malueg (forthcoming (b)), must be regular with $\widetilde{v}=0$ and $\hat{v}<\bar{v}$, when $c<\bar{v}$. Consequently, by Proposition 2 , it must be that $s(\widetilde{v})<0$, which is impossible. More generally, Proposition 2 shows how the assumption $\underline{v}=0$ may prevent regular equilibria of the subscription game from achieving specific theoretical bounds. This will be relevant for our analysis in Section 4. In this sense then, the assumption $\underline{v}=0$ is not a harmless normalization.

\section{Piecewise-linear equilibria}

The equilibrium characterization of the previous section is fairly general. Even if step-function equilibria are excluded by requiring $s$ to be regular, a vast multiplicity of equilibria results because the restrictions on the initial conditions $\widetilde{v}$ and $s(\widetilde{v})$ in Proposition 1 are weak. ${ }^{10}$ Indeed, $\widetilde{v}$ may be larger than $\underline{v}$ and, even when $\widetilde{v}$ equals $\underline{v}, s(\widetilde{v})$ is not uniquely determined except when $\underline{v}$ is zero. Moreover, in contrast to equilibrium derivation in the contribution game (see Barbieri and Malueg, forthcoming (a)), to describe all regular equilibria it is not sufficient to truncate and translate a baseline functional form. For example, as shown by Laussel and Palfrey (2003), for a given threshold $t$, different initial conditions yield different functional forms for different equilibria, even when valuations are uniformly distributed. Little progress seems possible without further simplifications. For this reason, we next turn to a special family of regular symmetric equilibria, those that are piecewise-linear. In particular, for $v \in[\widetilde{v}, \hat{v}]$ strategy $s$ will be linear and strictly increasing, while on $[\hat{v}, \bar{v}]$ it is constant. ${ }^{11}$ For their eminent tractability, linear strategies have often been sought as a first step in the analysis of complex situations. More importantly, as we shall see, in our framework regular symmetric piecewise-linear equilibria have another desirable feature: they can maximize the collector's utility.

\footnotetext{
${ }^{10}$ Such restrictions become stronger when one focuses on regular equilibria with $0<s^{\prime}(v)<\infty$, as Martimort and Moreira (2007) show.

${ }^{11}$ By Proposition $1, s$ is differentiable on $(\widetilde{v}, \hat{v})$. So a piecewise-linear continuous strategy must have constant slope on this interval.
} 


\subsection{Admissible distribution functions}

Where $s$ is linear, equations (1) and (2) simplify considerably. The following lemmas characterize the class of distribution functions for which a regular symmetric piecewise-linear equilibrium strategy $s$ can exist. For these distributions, the subsequent propositions show exactly when such equilibria exist, and in these cases they specify equilibrium strategies.

Lemma 1. A regular symmetric piecewise-linear equilibrium exists only if the inverse hazard rate $(1-F) / f$ is linear on $[\widetilde{v}, \hat{v}]$.

Proof. If $s$ is linear on $[\widetilde{v}, \hat{v}]$, that is, $s(v)=\alpha v+\beta$, then the definition of $G$ and the boundary condition $G(\widetilde{v})=\hat{v}$ imply $G(v)=\hat{v}+\widetilde{v}-v$, so we can simplify (1) as $\alpha(1-F(G(v)))-(v-(\alpha v+\beta)) f(G(v))=0$, which upon rearrangement becomes

$$
\frac{1-F(G(v))}{f(G(v))}=\left(\frac{1-\alpha}{\alpha}\right) v-\frac{\beta}{\alpha}
$$

Using $G(G(v))=v$, we obtain

$$
\frac{1-F(v)}{f(v)}=\left(\frac{1-\alpha}{\alpha}\right)(\hat{v}+\widetilde{v}-v)-\frac{\beta}{\alpha},
$$

so that on $[\widetilde{v}, \hat{v}]$ the inverse hazard rate is indeed linear.

Lemma 1 restricts $F$ only on $[\widetilde{v}, \hat{v}]$, the interval where (1) holds (cf. footnote 6 ). If we require linearity of the inverse hazard rate over the whole interval $[\underline{v}, \bar{v}]$, simple integration yields the following lemma, whose proof is in the Appendix.

Lemma 2. If the inverse hazard rate of $F$ is linear on $[\underline{v}, \bar{v}]$, then $F$ is either

$$
F(v)=1-e^{-r(v-\underline{v})}, \text { with } r>0 \text { and } \bar{v}=+\infty
$$

or

$$
F(v)=1-\left(\frac{\bar{v}-v}{\bar{v}-\underline{v}}\right)^{r} \text {, with } r>0 \text { and } \bar{v}<\infty .
$$

The cdf given by (6) is simply an exponential distribution with support $[\underline{v}, \infty)$; the cdf given by (7) we refer to as a "reverse power function" distribution. ${ }^{12}$

\footnotetext{
${ }^{12}$ To understand this terminology, consider the cdf on $[0,1]$ given by $\Psi(v)=v^{r}$, where $r>0$. This is often called a power function distribution, with density $\psi(v)=r v^{r-1}$. For the distribution $F$ in $(7)$ with support $[0,1]$, the variable $1-v$ has the density function $\psi$; that is, $f(v)=\psi(1-v)$.
} 
The following propositions, with proofs in the Appendix, apply Proposition 1 to characterize regular piecewise-linear symmetric equilibria for the distributions of Lemma 2. The next proposition shows for exponential distributions that regular piecewise-linear equilibria are particularly simple.

Proposition 3 (Equilibria for exponential distributions). Suppose $F$ is defined on $[\underline{v}, \infty)$ by $F(v)=1-$ $e^{-r(v-\underline{v})}$, where $r>0$. A regular piecewise-linear symmetric equilibrium exists if and only if $\underline{v} \geq 1 / r$ and $t \geq 2(\underline{v}-1 / r)$. In this case, $\widetilde{v}=\underline{v}$ and the regular piecewise-linear symmetric equilibrium strategy is given by

$$
s(v)= \begin{cases}s(\hat{v}) & \text { if } \hat{v}<v \leq \bar{v} \\ v-\frac{1}{r} & \text { if } \underline{v} \leq v \leq \hat{v}\end{cases}
$$

where $\hat{v}=t-\underline{v}+2 / r$.

Note that by Proposition 3 a piecewise-linear equilibrium exists for the exponential distribution only if $\underline{v}>0$, showing the frequent assumption that the support of players' values begins at 0 is not without loss of generality.

For the reverse power function distribution, the description of equilibria is slightly more involved. For clarity, we distinguish between the high-threshold case in Proposition 4 and the low-threshold case in Proposition 5 .

Proposition 4 (Equilibria for reverse power function distributions, high-threshold case). Suppose $F$ is defined on $[\underline{v}, \bar{v}]$ by $F(v)=1-\left(\frac{\bar{v}-v}{\bar{v}-\underline{v}}\right)^{r}$, where $r>0$ and $0 \leq \underline{v}<\bar{v}<\infty$, and assume

$$
t>\frac{(2+r) \underline{v}+r \bar{v}}{1+r}
$$

A regular piecewise-linear symmetric equilibrium exists if and only if $t<2 \bar{v}$. The regular piecewise-linear symmetric equilibrium strategy is given by

$$
s(v)= \begin{cases}\frac{r}{1+r} v+\frac{\widetilde{v}}{1+r} & \text { if } \widetilde{v} \leq v \leq \bar{v} \\ 0 & \text { if } \underline{v} \leq v<\widetilde{v},\end{cases}
$$

where $\widetilde{v}=\frac{t(1+r)-r \bar{v}}{2+r} \in(\underline{v}, \bar{v})$.

According to Proposition 4, when the threshold is high and players use piecewise-linear strategies, only when a player's value is sufficiently large does he have a chance of receiving the good, and even then is not assured of enjoying it. In contrast, the next proposition shows that for a low threshold, when using 
piecewise-linear strategies all players have a chance of enjoying the good, and those with sufficiently high values are assured they will. Thus, the low-cost case is similar to that of the exponential distribution.

Proposition 5 (Equilibria for reverse power function distributions, low-threshold case). Suppose $F$ is defined on $[\underline{v}, \bar{v}]$ by $F(v)=1-\left(\frac{\bar{v}-v}{\bar{v}-\underline{v}}\right)^{r}$, where $r>0$ and $0 \leq \underline{v}<\bar{v}<\infty$, and assume

$$
t \leq \frac{(2+r) \underline{v}+r \bar{v}}{1+r}
$$

A regular piecewise-linear symmetric equilibrium exists if and only if

$$
t \geq \frac{r}{1+r}(\bar{v}-\underline{v}(2+r))
$$

and

$$
t \geq-\frac{2}{1+r}(\bar{v}-\underline{v}(2+r))
$$

The regular piecewise-linear symmetric equilibrium strategy is given by

$$
s(v)= \begin{cases}s(\hat{v}) & \text { if } \hat{v}<v \leq \bar{v} \\ \frac{r}{1+r} v-\frac{\bar{v}-\hat{v}-\underline{v}}{1+r} & \text { if } \underline{v} \leq v \leq \hat{v}\end{cases}
$$

where $\hat{v}=\frac{t(1+r)+2 \bar{v}}{2+r}-\underline{v} \in(\underline{v}, \bar{v})$.

Though there typically exist multiple equilibria in the subscription game, we have focused on the piecewise-linear regular equilibria as especially attractive. However, it may be that in some cases they do not exist. Nevertheless, we next show that when they do exist they can maximize the seller's expected utility over all incentive compatible mechanisms. Additionally, for the exponential and reverse power function distributions, we show that when such equilibria do not exist, the subscription game is not a mechanism that can maximize the utility of the collector.

\section{Optimal provision of a discrete public good: an application of linear equilibrium strategies}

We begin this section by allowing the collector the choice of any incentive compatible and individually rational mechanism. We will then show how, when regular piecewise-linear equilibria exist, a slight modification of the subscription game implements the allocation that maximizes the collector's utility. 


\subsection{The utility-maximizing direct mechanism}

The seller's utility-maximization problem can be solved adapting Myerson's (1981) optimal auction design. By the Revelation Principle it suffices to consider only direct mechanisms that are feasible: both incentive compatible and individually rational. Direct mechanisms are triples of functions $\left(p, x_{1}, x_{2}\right)$ defined on $[\underline{v}, \bar{v}] \times$ $[\underline{v}, \bar{v}]$. Players simultaneously report values $v_{1}^{\prime}$ and $v_{2}^{\prime}$; then $x_{i}\left(v_{1}^{\prime}, v_{2}^{\prime}\right)$ is player $i$ 's payment to the seller and $p\left(v_{1}^{\prime}, v_{2}^{\prime}\right) \in[0,1]$ is the probability the good is provided. The only difference with Myerson (1981) is that $p$ is not indexed by players: we are dealing with a pure public good. Moreover, we allow the seller to attach weight $\rho \in[0,1)$ to the contributors' payoffs. When $\rho \in(0,1)$, the seller is in part altruistic, taking into account not only his profit, but also the players' utility. In sum, the collector's problem is to maximize

$\int_{\underline{v}}^{\bar{v}} \int_{\underline{v}}^{\bar{v}}\left\{\left[x_{1}\left(v_{1}, v_{2}\right)+x_{2}\left(v_{1}, v_{2}\right)-c p\left(v_{1}, v_{2}\right)\right]+\rho\left[\left(v_{1}+v_{2}\right) p\left(v_{1}, v_{2}\right)-x_{1}\left(v_{1}, v_{2}\right)-x_{2}\left(v_{1}, v_{2}\right)\right]\right\} d F\left(v_{1}\right) d F\left(v_{2}\right)$ over all feasible mechanisms.

Cornelli (1996) analyzed this problem with $\rho=0$, that is, for a profit-maximizing seller. A straightforward adaptation of Cornelli's argument yields the following.

Lemma 3 (Cornelli, 1996). If $w(v) \equiv v-(1-\rho) \frac{1-F(v)}{f(v)}$ is increasing on $[\underline{v}, \bar{v}]$, then a utility-maximizing feasible direct mechanism $\left(p, x_{1}, x_{2}\right)$ has

$$
p\left(v_{1}, v_{2}\right)= \begin{cases}1 & \text { if } w\left(v_{1}\right)+w\left(v_{2}\right) \geq c \\ 0 & \text { if } w\left(v_{1}\right)+w\left(v_{2}\right)<c .\end{cases}
$$

Next we connect a player's conditional expected payment to the function $p$ in a feasible mechanism. Assuming truthful revelation by player 2, player 1's expected payoff when reporting $v_{a}$ is

$$
U_{1}\left(v_{a} \mid v_{1}\right)=\mathrm{E}\left[v_{1} p\left(v_{a}, v_{2}\right)-x_{1}\left(v_{a}, v_{2}\right)\right]=v_{1} P_{1}\left(v_{a}\right)-X_{1}\left(v_{a}\right)
$$

where

$$
P_{1}\left(v_{a}\right)=\int_{\underline{v}}^{\bar{v}} p\left(v_{a}, v_{2}\right) d F\left(v_{2}\right) \quad \text { and } \quad X_{1}\left(v_{a}\right)=\int_{\underline{v}}^{\bar{v}} x_{1}\left(v_{a}, v_{2}\right) d F\left(v_{2}\right) .
$$

Analogous formulas apply for player 2. In the truth-telling equilibrium, conditional on $v_{i}, P_{i}\left(v_{i}\right)$ is player $i$ 's perceived probability the good will be provided and $X_{i}\left(v_{i}\right)$ is his expected payment to the seller. Let $U_{i}^{*}\left(v_{i}\right) \equiv U_{i}\left(v_{i} \mid v_{i}\right)$ be player $i$ 's payoff in the truth-telling equilibrium of a feasible direct mechanism. 
Incentive compatibility requires

$$
0=\left.\frac{\partial U_{i}\left(v_{a} \mid v_{i}\right)}{\partial v_{a}}\right|_{v_{a}=v_{i}}=v_{i} P_{i}^{\prime}\left(v_{i}\right)-X_{i}^{\prime}\left(v_{i}\right)
$$

$\mathrm{SO}$

$$
X_{i}^{\prime}\left(v_{i}\right)=v_{i} P_{i}^{\prime}\left(v_{i}\right)
$$

The Envelope Theorem gives $d U_{i}^{*}\left(v_{i}\right) / d v_{i}=P_{i}\left(v_{i}\right)$, implying $U_{i}^{*}$ is nondecreasing. Therefore, an incentive compatible mechanism is individually rational if and only if $U_{i}^{*}(\underline{v}) \geq 0$. As Myerson (1981) and Cornelli (1996) show, seller's utility maximization requires this constraint to bind. The utility-maximizing conditional expected payment can be found by integrating (14) with the boundary condition ensuring $U_{i}^{*}(\underline{v})=0$. Expected cost is $c$ times the probability the good is provided.

\subsection{Utility maximization in the subscription game}

For implementation purposes, we slightly modify the standard subscription game. First, with binding commitment, the collector announces the threshold $t$ and an individual's non-refundable entry fee $\varphi$. Next, after observing the realization of their own private values, contributors independently and simultaneously decide whether to pay $\varphi$. If either does not pay the entry fee, the game ends. If both pay $\varphi$, the game moves to the third stage, in which contributors play the standard subscription game: players simultaneously contribute any amount; these contributions are refunded if they total less than $t$ but otherwise are retained by the collector. Only in the latter case does the collector provide the good, incurring cost $c$.

The next propositions show that, when regular piecewise-linear regular equilibria exist, the collector can choose $t$ and $\varphi$ so that his utility in the modified subscription game coincides with his utility in the optimal direct mechanism described in Section 4.1. In turn, we will reference the necessary and sufficient conditions in Propositions 3, 4, and 5. Given threshold $t$, we will take the corresponding equilibria described in Propositions 3, 4, and 5 to be the continuation equilibria after players pay the entry fee $\varphi$. We first consider the exponential distribution in (6). Because $w(v)=v-(1-\rho) / r$ is increasing in $v$, Lemma 3 applies. Straightforward calculations show that when $c \leq 2(\underline{v}-(1-\rho) / r)$ the collector optimally provides the public good with probability one, and charges $2 \underline{v}$. This can be easily accomplished in our modified subscription game by setting the entry fee $\varphi=\underline{v}$ and the threshold $t=0$. For higher costs, the following proposition shows how the collector obtains the same utility as in the optimal direct mechanism.

Proposition 6 (Utility maximization: exponential distributions). Suppose $F$ is defined on $[\underline{v}, \infty)$ by $F(v)=$ $1-e^{-r(v-\underline{v})}$, where $r>0$ and $\underline{v} \geq 1 / r$. Assume further that $c>2(\underline{v}-(1-\rho) / r)$. The collector can obtain 
the same utility as in the optimal direct mechanism in Section 4.1 by setting the contribution threshold at $t=c-2 \rho / r$ and the nonrefundable entry fee at $\varphi=e^{-2(1-\rho)-c r+2 \underline{v} r} / r$. The continuation equilibrium is described in (8).

Our proof uses Myerson's (1981) Revenue Equivalence Theorem. For given $c$, if $t$ is chosen so the provision region in the subscription game matches that in the corresponding optimal mechanism, then the probability a player obtains the good conditional on his value $\left(P_{i}\left(v_{i}\right)\right.$ in the notation of Section 4.1$)$ is the same in both settings. ${ }^{13}$ By Myerson's result, each player's expected payments will then be the same in both regimes if the player with value $\underline{v}$ earns the same conditional expected payoff (namely 0 ) in both settings. We set the entry fee $\varphi$ to make this so, leaving players willing to pay $\varphi$. Because provision regions coincide, expected costs are identical.

Proof of Proposition 6. As noted above, $w(v)=v-(1-\rho)(1-F(v)) / f(v)=v-(1-\rho) / r$, so the utilitymaximizing direct mechanism provides the good exactly where $v_{1}+v_{2} \geq c+2(1-\rho) / r$.

Now consider the provision region for the regular equilibrium of Proposition 3. As given in $(8), s(v)=$ $v-1 / r$ for all $v \leq \hat{v}=t-\underline{v}+2 / r$, and the good is provided if and only if $v_{1}+v_{2} \geq t+2 / r$. Therefore, to match the two provision regions, the subscription game threshold is set at $t=c-2 \rho / r$. If no entry fee were required, the payoff to the lowest type would be

$$
(\underline{v}-s(\underline{v})) \operatorname{Pr}(v>\hat{v})=\frac{1}{r} e^{-r(\hat{v}-\underline{v})}=\frac{1}{r} e^{-2(1-\rho)+2 r \underline{v}-r c} .
$$

Therefore, to extract all surplus from the lowest-value type, the entry fee is set to $e^{-2(1-\rho)-c r+2 \underline{v} r} / r$.

It is worth noting that Proposition 6 covers the case $2(\underline{v}-(1-\rho) / r)<c \leq 2 \underline{v}$, where a fully efficient equilibrium of the subscription game exists. Indeed, because $c \leq 2 \underline{v}$, the contributors' asymmetric information is irrelevant for efficiency purposes: it is common knowledge that the good should always be provided and both contributors are willing to contribute $c / 2$ regardless of their private information. Nonetheless, as long as $\rho<1$, the collector finds it more profitable to restrict the probability of provision as described in Proposition 6 . This behavior of the collector is completely analogous to a monopolist restricting the quantity supplied to increase the price charged, or to the imposition of a reserve price in optimal auctions.

While for the exponential distribution piecewise-linear regular equilibria do not exist when $\underline{v}<1 / r$, there may well exist nonlinear equilibria continuous on $[\widetilde{v}, \hat{v}]$. Nevertheless, no such equilibria of the (modified)

\footnotetext{
${ }^{13}$ It is now clear, too, why step-function equilibria cannot be utility-maximizing. The "southwest" boundary of the region of optimal provision is where $w\left(v_{1}\right)+w\left(v_{2}\right)=c$. If $w(\cdot)$ is strictly increasing, then this boundary will be strictly decreasing and continuous in $v_{1} \times v_{2}$-space. Step-function equilibria give a boundary that is not strictly decreasing.
} 
subscription game yield the same utility as the optimal mechanism. Thus, without restricting attention to linear strategies, the following proposition provides a converse to the previous result.

Proposition 7. Suppose $F$ is defined on $[\underline{v}, \infty)$ by $F(v)=1-e^{-r(v-\underline{v})}$, where $r>0$ and $\underline{v}<1 / r$. For any continuation equilibrium of the subscription game, the collector cannot obtain the same utility as in the optimal direct mechanism in Section 4.1.

Proof. Applying Lemma 3, we obtain that the provision region in the optimal mechanism is $v_{1}+v_{2} \geq c+2 / r$. If an equilibrium of the subscription game can match this provision region it must have $G^{\prime}(v)=-1$, so $G(v)=k_{1}-v$ for some constant $k_{1}$, where $G$ is defined in Proposition 1. Equation (1) then reduces to

$$
s^{\prime}(v)=r(v-s(v))
$$

a solution to this differential equation must have the form

$$
s(v)=v-\frac{1}{r}+k_{2} e^{-r v}
$$

for some constant $k_{2}$. When $k_{2}=0$ one obtains our earlier candidate for a linear-strategy equilibrium. However, because $\underline{v}<1 / r$, Proposition 3 implies this is not an equilibrium. When $k_{2} \neq 0$, constancy of $s(v)+s(G(v))$ on $[\widetilde{v}, \hat{v}]$ implies that, for some constant $k_{3}$,

$$
k_{1}-\frac{2}{r}+k_{2}\left(e^{-r v}+e^{-r\left(k_{1}-v\right)}\right)=k_{3}
$$

differentiation with respect to $v$ implies $e^{-r v}=e^{-r\left(k_{1}-v\right)}$, which cannot be satisfied for an interval of $v$ 's. Hence, there is no equilibrium with $k_{2} \neq 0$.

Combining Propositions 6 and 7, the following corollary provides necessary and sufficient conditions for the subscription game to maximize the collector's utility when values have an exponential distribution.

Corollary 1. Suppose $F$ is defined on $[\underline{v}, \infty)$ by $F(v)=1-e^{-r(v-\underline{v})}$, where $r>0$. Assume further that $c>2(\underline{v}-(1-\rho) / r)$. Through the modified subscription game the collector can obtain the same utility as in the optimal direct mechanism if and only if $\underline{v} \geq 1 / r$.

We now consider reverse power function distributions. Lemma 3 again applies, and straightforward calculations show that when $\mathrm{cr} \leq 2(((1-\rho)+r) \underline{v}-(1-\rho) \bar{v})$ (the low-cost case), the collector optimally provides the public good with probability one and charges $2 \underline{v}$ - there are many ways to implement this allocation via the subscription game. For $c r>2(((1-\rho)+r) \underline{v}-(1-\rho) \bar{v})$ it is convenient to distinguish two 
cases. We begin with the high-cost case. (The proofs of the remaining propositions follow the logic of those of Propositions 6 and 7 and can be found in the Appendix.)

Proposition 8 (Utility maximization: reverse power distributions, high cost). Suppose $F$ is defined on $[\underline{v}, \bar{v}]$ by $F(v)=1-\left(\frac{\bar{v}-v}{\bar{v}-\underline{v}}\right)^{r}$, where $r>0$ and $0 \leq \underline{v}<\bar{v}<\infty$. Assume further that c satisfies $\underline{v}((1-\rho)+r)+$ $(r-(1-\rho)) \bar{v} \leq c r<2 \bar{v} r$. The collector can obtain the same utility as in the optimal direct mechanism in Section 4.1 by setting the contribution threshold at

$$
t=\frac{c r(2+r)+2 \bar{v}(1-\rho(1+r))}{(1+r)((1-\rho)+r)}
$$

and the nonrefundable entry fee at $\varphi=0$. The continuation equilibrium is described in (10).

Observe that in the high-cost case, the unmodified subscription game is optimal - an entry fee need not be charged. This is because types near $\underline{v}$ have no chance of obtaining the good and therefore earn zero surplus. For lower costs of production the seller can be expected to set a lower threshold; once the type- $\underline{v}$ contributor has a chance of obtaining the good, an entrance fee must be added to ensure this contributor is left with no surplus. Nevertheless, turning to the medium-cost case, we note that the functional form of the optimal threshold is the same as in the high-cost case.

Proposition 9 (Utility maximization: reverse power distributions, medium cost). Suppose $F$ is defined on $[\underline{v}, \bar{v}]$ by $F(v)=1-\left(\frac{\bar{v}-v}{\bar{v}-\underline{v}}\right)^{r}$, where $r>0$ and $0 \leq \underline{v}<\bar{v}<\infty$. Assume further that $c$ satisfies $2(((1-\rho)+r) \underline{v}-(1-\rho) \bar{v})<c r \leq \underline{v}((1-\rho)+r)+(r-(1-\rho)) \bar{v}$. If either

a. $\bar{v}-\underline{v}(2+r) \leq 0 ;$ or

b. $\bar{v}-\underline{v}(2+r)>0$ and $c r \geq \bar{v}(r+\rho-1)-\underline{v} r(1+r-\rho)$,

then the collector can obtain the same utility as in the optimal direct mechanism in Section 4.1 by setting the contribution threshold at

$$
t=\frac{c r(2+r)+2 \bar{v}(1-\rho(1+r))}{(1+r)((1-\rho)+r)}
$$

and the nonrefundable entry fee at

$$
\varphi=\frac{[\bar{v}(r-1+\rho)+\underline{v}(1+r-\rho)-c r]^{r+1}}{(1+r)^{r+2}(\bar{v}-\underline{v})^{r}} .
$$

The continuation equilibrium is described in (12).

Our analysis has implications for the study by Alboth et al. (2001), who consider a seller of a discrete public good facing two contributors whose individual values are independently and uniformly distributed 
over $[0,1]$. The seller's cost of production is zero. Setting a threshold $t$ that total contributions must reach before the good is provided, the seller expects to earn positive revenue in the Bayesian equilibrium of the subscription game. Given $t<2$, Alboth et al. derive the unique symmetric equilibrium in which a player's strategy is a strictly increasing, continuously differentiable function of his own value, having range $[0, t]$. (Thus, every equilibrium they derive has $\widetilde{v}=0$ and $\hat{v}=1$.) Varying threshold $t$ they seek the corresponding equilibrium maximizing expected revenue; revenue is so maximized at $t=1 / 2$, achieving value $1 / 3$; the associated equilibrium strategy is $s(v)=v / 2$ for all $v \in[0,1]$. Their analysis, however, leaves unanswered three important questions:

1. Do other subscription game equilibria yield greater revenue?

2. Do other selling mechanisms yield greater revenue?

3. If $c>0$ can any of Alboth et al.'s strictly increasing equilibria be profit-maximizing for the seller?

Our analysis implies the answer to all three questions is No. Propositions 8 covers the case where values are uniformly distributed over $[0,1](\underline{v}=0, \bar{v}=1, r=1)$. If $\rho=0$ and $c=0$ (the case of Alboth et al.), then the subscription game yields the profit of the optimal mechanism when the threshold is set at $t=1 / 2$, the entry fee is $\varphi=0$, and players use strategy $s(v)=v / 2$. Thus, not only is the equilibrium Alboth et al. derive profit-maximizing in the subscription game, no other mechanism yields greater profit. For $t \neq 1 / 2$ the equilibria Alboth et al. derive are highly nonlinear and apparently give provision regions without the linear boundary found in an optimal mechanism, and therefore would appear not to maximize the seller's profit over all incentive compatible mechanisms. ${ }^{14}$ To see this is indeed the case, note that in the equilibria of Alboth et al., where strategies are strictly increasing throughout, all types in $(0,1]$ have a strictly positive probability of obtaining the good. However, for $\rho=0$, so that the seller maximizes profits, and $c \in(0,2)$, the optimal mechanism specifies that players with value $v \in[0, c / 2)$ have zero chance of obtaining the good. ${ }^{15}$ Thus, the provision regions of the optimal mechanism and the equilibria of Alboth et al. do not match, implying by the Revenue Equivalence Theorem that the revenues are not equal.

Analogous to Proposition 7, our final proposition for the subscription game provides for the reverse power function distribution the same kind of converse result to the optimality of linear strategies: if linear strategies do not exist, then the (modified) subscription game cannot maximize the collector's utility.

Proposition 10. Suppose $F$ is defined on $[\underline{v}, \bar{v}]$ by $F(v)=1-\left(\frac{\bar{v}-v}{\bar{v}-\underline{v}}\right)^{r}$, where $r>0$ and $0 \leq \underline{v}<\bar{v}<\infty$. Assume further that $2(((1-\rho)+r) \underline{v}-(1-\rho) \bar{v}) \leq c r \leq \underline{v}((1-\rho)+r)+(r-(1-\rho)) \bar{v}$. If $\bar{v}-\underline{v}(2+r)>0$

\footnotetext{
${ }^{14}$ Similarly, with a uniform distribution of values, the contribution game cannot be optimal (even when $c=0$, the case of Alboth et al.) as the continuous equilibria have a provision region bounded by a hyperbola (Barbieri and Malueg, forthcoming (a)).

${ }^{15}$ To see this, recall that for values uniformly distributed over $[0,1]$ and $\rho=0$, the optimal mechanism provides the good if and only if $v_{1}+v_{2} \geq 1+c / 2$.
} 
and $\mathrm{cr}<\bar{v}(r+\rho-1)-\underline{v} r(1+r-\rho)$, then, for any continuation equilibrium of the subscription game, the collector cannot obtain the same utility as in the optimal direct mechanism in Section 4.1.

Combining Propositions 8-10, the following corollary provides necessary and sufficient conditions for the subscription game to maximize the collector's utility when values have a reverse power function distribution.

Corollary 2. Suppose $F$ is defined on $[\underline{v}, \bar{v}]$ by $F(v)=1-\left(\frac{\bar{v}-v}{\bar{v}-\underline{v}}\right)^{r}$, where $r>0$ and $0 \leq \underline{v}<\bar{v}<\infty$. Assume further that $2(((1-\rho)+r) \underline{v}-(1-\rho) \bar{v}) \leq c r \leq 2 r \bar{v}$. Through the modified subscription game the collector can obtain the same utility as in the optimal direct mechanism if and only if $\bar{v} \leq(2+r) \underline{v}$ or $c r \geq \bar{v}(r+\rho-1)-\underline{v} r(1+r-\rho)$.

The results of this section display natural comparative statics properties. One may easily verify that the optimal threshold $t$ is increasing in the cost $c$ and decreasing in the altruism parameter $\rho$. To understand this note than an increase in $c$ requires a smaller provision region, which is achieved in the subscription game with an increase in $t$. An increase in $\rho$ gives greater weight to players' welfare, and intuitively the provision region should be increased; indeed, the optimal provision region expands and this is achieved with a reduction in $t$. The results for the optimal entry fee $\varphi$ have the opposite sign of those for $t$. They are driven by the requirement $U_{i}^{*}(\underline{v})=0$ in Section 4.1. Indeed, a smaller threshold $t$ implies a larger utility for type $\underline{v}$, so that the entry fee must increase to ensure that the utility of the lowest type remains zero. It is worth mentioning that, when $\rho=0$, the collector's ex post profit is positive: The optimal threshold is always larger than the cost of production $c$. Intuitively, as $\rho$ increases, the collector's profit becomes negative for some combinations of valuations: The collector becomes itself a contributor to the public good. Indeed, even the ex ante expected profit becomes negative as $\rho$ grows large, as implied by the Myerson-Satterthwaite theorem. For valuations uniformly distributed on $[0,1]$, one can show that in the high-cost case $(\rho<c<2)$ the collector's ex post profits are always nonnegative for $\rho \leq 1 / 2$ and ex ante expected profits are positive as long as $\rho<2 / 3$.

\subsection{Utility maximization in the contribution game}

Our analysis so far has shown that, for the family of distribution functions characterized by a linear inverse hazard rate, the subscription game admits equilibria that maximize the collector's utility. Importantly, this result holds for an interval of provision costs $c$ and for all altruism parameters $\rho \in[0,1)$. A natural question is whether a similar result can be obtained for the closely related contribution game. A negative answer would provide a meaningful comparison of the contribution and subscription games, additional to the existing efficiency comparisons in the literature, that are not conclusive. Indeed, Menezes et al. (2001) show classical inefficiency of both subscription and contribution games. Moreover, Laussel and Palfrey (2003) and Barbieri 
and Malueg (forthcoming (a)) show interim incentive efficiency for both subscription and contribution games, respectively, albeit only for a uniform distribution of valuations and for different ranges of $c$.

Note first that Lemma 3 implies that we can restrict attention to continuous and symmetric equilibria of the contribution game in our quest to match the optimal provision region, as discussed above (cf. footnote 13). From Barbieri and Malueg (forthcoming (a)) one can then immediately see how the choice of the contribution game cannot maximize the seller's utility if either $\underline{v}=0$ or $w(\underline{v})+w(\bar{v})<c$. In the first case, under the assumption $f(\underline{v})>0$, no continuous equilibrium exists. In the second, the optimal provision region specifies that types close to $\underline{v}$ should receive the public good with probability zero. This condition is inconsistent with existence of a continuous equilibrium of the contribution game.

A closer inspection of the characterization result in Proposition 1 in Barbieri and Malueg (forthcoming (a)) yields the following additional necessary condition, with proof in the Appendix, for the contribution game to maximize the utility of a seller with altruism parameter $\rho$.

Proposition 11 (Utility maximization: contribution game). Assume $f$ is strictly positive on $[\underline{v}, \bar{v})$ and consider some $c_{m}>0$ and some $\epsilon>0$. Suppose that, for all $c \in\left(c_{m}-\epsilon, c_{m}+\epsilon\right)$, there exists an equilibrium of the contribution game such that a seller with altruism parameter $\rho$ receives the same utility as in the optimal direct mechanism in Section 4.1. Then the distribution function $F(v)$ solves

$$
\frac{w^{\prime \prime}(v)}{w^{\prime}(v)}=\frac{f^{\prime}(v)}{f(v)}-\frac{1}{v}
$$

for all $v \in\left(w^{-1}\left(\frac{c_{m}-\epsilon}{2}\right), w^{-1}\left(\frac{c_{m}+\epsilon}{2}\right)\right)$, where $w$ is defined in Lemma 3 .

The differential equation in Proposition 11 may be used to show that, for the distribution functions in Lemma 2, the contribution game is never optimal for the seller, in contrast to the subscription game. More importantly, equation (15) shows that the distribution function for which the contribution game may be optimal depends on the specific value of $\rho$, as the following lemma, with proof in the Appendix, shows.

Proposition 12. There exists no distribution function $F$ independent of $\rho$ and satisfying the assumptions of Section 4.1, namely $w^{\prime}(v)>0$, such that $F$ solves (15) for an open set of $\rho$ 's.

Therefore, as soon as one requires optimality not just for a single value of the altruism parameter, as reasonable in judging the properties of mechanisms like the contribution or subscription games, Proposition 11 implies that the contribution game cannot be optimal. This is a sharp contrast with the subscription game, as demonstrated in the previous section. The difference stems from the different treatment of insufficient contributions. The full-refund feature of the subscription game generates a much greater latitude for the collector. Indeed, through the careful choice of the threshold $t$ described in the previous section, different 
optimal provision regions induced by changes in the cost $c$ and by different values of $\rho$ may be jointly accommodated in the subscription game. On the contrary, because insufficient contributions are not refunded, equilibria are more tightly determined in the contribution game, especially if continuity of equilibrium strategies is required. Therefore, the contribution game is a far less flexible mechanism for the collector than is the contribution game. To understand how the no-refund feature limits the flexibility of the collector, it is enough to consider the result, established in Barbieri and Malueg (forthcoming (a)), that all continuous equilibria of the contribution game must have $\widetilde{v}=\underline{v}$. If that were not the case, it would imply that the contributions of types $\underline{v}$ and $\bar{v}$ total less than the threshold $t$. By continuity of the equilibrium strategy then, there exists a player with value $v^{\prime} \in(\underline{v}, \widetilde{v})$ that contributes a strictly positive amount with no expectation of the project being completed. Therefore we obtain a contradiction to equilibrium, since type $v^{\prime}$ has a profitable deviation to a contribution of zero because insufficient contributions are not refunded. Similar restrictions can be derived for the slope of the contribution function, as Barbieri and Malueg (forthcoming (a)) and Menezes et al. (2001) show. Such restrictions are the key step in the proof Proposition 11.

\section{Conclusion}

In the symmetric subscription game, piecewise-linear regular equilibria exist for the exponential and reverse power function distributions of players' values. Moreover, if a regular symmetric equilibrium strategy is piecewise-linear, then the distribution of players' types must agree with an exponential or reverse power function distribution, at least over the interval $[\widetilde{v}, \hat{v}]$. For the exponential and reverse power function distributions we have characterized exactly when piecewise-linear regular equilibria exist, and we have shown such equilibria can be utility-maximizing for the seller. Moreover, for these distributions only piecewise-linear equilibria can maximize the seller's utility over all incentive compatible mechanisms. While we have shown the contribution game is, generically, suboptimal for the seller, it remains an open question whether, for other distributions of players' types, nonlinear equilibria of the subscription game (possibly with entry fees) can be optimal.

\section{Appendix}

Proof of Proposition 1. Necessity: Note that on $[\widetilde{v}, \hat{v}]$ the pairs $(v, G(v))$ represent the equilibrium completion frontier in $v_{1} \times v_{2}$-space. The payoff to a player with value $v \in[\widetilde{v}, \hat{v}]$ contributing $s\left(v_{a}\right) \in[s(\widetilde{v}), s(\hat{v})]$ is $U\left(s\left(v_{a}\right) \mid v\right)=\left(v-s\left(v_{a}\right)\right)\left(1-F\left(G\left(v_{a}\right)\right)\right)$; since $s(v)$ is optimal it must satisfy the following first-order 
optimization condition almost everywhere

$$
s^{\prime}(v)(1-F(G(v)))+(v-s(v)) f(G(v)) G^{\prime}(v)=0
$$

which is (1). Differentiating the defining equation for $G$, we obtain almost everywhere $s^{\prime}(v)+s^{\prime}(G(v)) G^{\prime}(v)=$ 0 , which can be rearranged to give (2). To show that (1) and (2) must hold everywhere on $(\widetilde{v}, \hat{v})$, we use $G(G(v))=v$ and rearrange (1) and (2) as

$$
s^{\prime}(v)=h(v)(G(v)-s(G(v)))
$$

where $h(y) \equiv f(y) /(1-F(y))$ is the hazard rate function associated with $F$. Because $s, G$, and $h$ are continuous on $(\widetilde{v}, \hat{v})$ it follows that left-hand and right-hand derivatives of $s$ exist and are equal everywhere on $(\widetilde{v}, \hat{v})$ - thus, $s$ is differentiable on $(\widetilde{v}, \hat{v})$. Since contributions and payoffs in equilibrium must be positive, we need $0 \leq s(v) \leq v$, for $v \in[\widetilde{v}, \bar{v}]$.

If $\widetilde{v}>\underline{v}$, then two additional conditions must be satisfied. First, it is necessary that

$$
s(\widetilde{v})=\widetilde{v},
$$

for if $s(\widetilde{v})<\widetilde{v}$ then types in $[\underline{v}, \widetilde{v})$ sufficiently near $\widetilde{v}$ could profitably deviate to a contribution of $s(\widetilde{v})$. Second, it must be that

$$
(v-s(v))(1-F(G(v))) \geq \bar{v}-t \quad \forall v \geq \hat{v}
$$

so that players contributing the maximum cannot, by ensuring the project is provided, strictly increase their payoffs with a contribution of $t$ rather than $s(\hat{v})$.

Sufficiency: Now we show that conditions (1)-(4) suffice to characterize a regular symmetric equilibrium. To show that the first-order condition (1) actually identifies a best response, first observe that for any announcement $v_{a} \in(\widetilde{v}, \hat{v})$,

$$
\begin{aligned}
\frac{d U\left(s\left(v_{a}\right) \mid v\right)}{d v_{a}} & =-s^{\prime}\left(v_{a}\right)\left(1-F\left(G\left(v_{a}\right)\right)\right)-\left(v-s\left(v_{a}\right)\right) f\left(G\left(v_{a}\right)\right) G^{\prime}\left(v_{a}\right) \\
& =\left(v_{a}-v\right) f\left(G\left(v_{a}\right)\right) G^{\prime}\left(v_{a}\right)
\end{aligned}
$$

where the second equality follows from (1) evaluated at $v=v_{a}$. Because $G^{\prime}\left(v_{a}\right) \leq 0$, we have

$$
\frac{d U\left(s\left(v_{a}\right) \mid v\right)}{d v_{a}} \gtreqless 0 \text { for } v_{a} \lesseqgtr v
$$


implying that $U\left(s\left(v_{a}\right) \mid v\right)$ is quasiconcave in $v_{a} \in(\widetilde{v}, \hat{v})$, achieving a maximum at $s\left(v_{a}\right)=s(v)$. By continuity of $s$ (in $v$ ) and $U$ (in $s$ and $v$ ), $s$ is also optimal for $v=\widetilde{v}, \hat{v}$. Thus, we have shown that over the interval of contributions $[s(\widetilde{v}), s(\hat{v})], s(v)$ is optimal for all $v \in[\widetilde{v}, \hat{v}]$. It remains to show $s(v)$ is globally optimal for all $v \in[\widetilde{v}, \hat{v}]$. A contribution less than $s(\widetilde{v})$ implies the good will not be provided and the corresponding payoff is 0 ; hence, given (3), such a contribution is not strictly better than $s(v)$. A contribution in the interval $(s(\hat{v}), t)$ does not increase the probability of provision beyond contributing $s(\hat{v})$ as all types $v<\widetilde{v}$ contribute 0 . Therefore, the only other deviation from $s(v)$ to consider is $t$, which would ensure the good is provided. However, we see that, for any $v \in[\widetilde{v}, \hat{v}]$,

$$
\begin{array}{rlr}
U(s(v) \mid v) & \geq U(s(\hat{v}) \mid v) & \\
& =(v-s(\hat{v}))(1-F(G(\hat{v}))) & \\
& =(v-\hat{v})(1-F(G(\hat{v})))+(\hat{v}-s(\hat{v}))(1-F(G(\hat{v}))) & \\
& \geq(v-\hat{v})(1-F(G(\hat{v})))+(\hat{v}-t) & \text { (by condition }(4)) \\
& =(v-t)+(\hat{v}-v) F(G(\hat{v})) & \\
& \geq v-t,
\end{array}
$$

showing a deviation to $t$ is not profitable.

Turning attention to types in $(\hat{v}, \bar{v}]$, we show $s(v)=s(\hat{v})$ is optimal. There are two possibilities. The first is $\widetilde{v}=\underline{v}$. Here, because $s(\hat{v})+s(\widetilde{v})=s(\hat{v})+s(\underline{v})=t$ and $s$ is nondecreasing, a contribution $s(\hat{v})$ is enough to ensure provision of the good, so any larger contribution is dominated by $s(\hat{v})$. The second possibility is $\widetilde{v}>$ $\underline{v}$. In this case, condition (4) is sufficient to discourage a deviation to a contribution equal to $t$ by any type $v \in(\hat{v}, \bar{v}]$, and to any contribution in $(s(\hat{v}), t)$ because $s(v)=0$ for $v \in[\underline{v}, \widetilde{v})$. Finally, note that because $s(\hat{v})$ is optimal for type $\hat{v}$ and, as is easily shown, larger types will want to contribute at least as much as type $\hat{v}$, deviations to levels below $s(\hat{v})$ are not profitable for types $v \in(\hat{v}, \bar{v}]$.

As for types in $[\underline{v}, \widetilde{v})$, they can exist only when $\widetilde{v}>\underline{v}$. In this case, $s(\widetilde{v})=\widetilde{v}$ by condition (4) so types in $[\underline{v}, \widetilde{v})$ find it optimal to contribute any amount less than $s(\widetilde{v})$, in order to avoid triggering completion with some positive probability and thereby realizing a strictly negative payoff. Therefore, we can set their contributions to zero. This establishes that $s(v)$ is optimal for all $v$.

Proof of Proposition 2. By contradiction assume that, for some $t>0,(s, s)$ is a regular equilibrium with $s(\widetilde{v})=\widetilde{v}$ and $\hat{v}<\bar{v}$. Then, it must satisfy the conditions in Proposition 1. Define $G:[\widetilde{v}, \hat{v}] \rightarrow[\widetilde{v}, \hat{v}]$ as in Proposition 1. Then $G$ is strictly decreasing and continuous, with $G(\widetilde{v})=\hat{v}$ and $G(\widetilde{v})=\hat{v}$. After algebraic 
manipulations and using $G(G(v))=v$, conditions (1) and (2) may be written as

$$
s^{\prime}(v)=h(v)(G(v)-s(G(v)))
$$

and

$$
G^{\prime}(v)=-\frac{h(v)}{v-s(v)} \frac{G(v)-s(G(v))}{h(G(v))}
$$

where $h(y) \equiv f(y) /(1-F(y))$ is the hazard rate function associated with $F$. Define $v_{m}$ as the type that contributes $t / 2: v_{m} \equiv s^{-1}(t / 2)$; note that in a regular equilibrium $v_{m}$ exists and $v_{m} \in(\widetilde{v}, \hat{v})$. Fix any $v \in\left[\widetilde{v}, v_{m}\right]$ and observe that $G(v) \in\left[v_{m}, \hat{v}\right]$. Define $m$ as

$$
m \equiv \min _{v \in\left[v_{m}, \hat{v}\right]} \frac{v-s(v)}{h(v)} .
$$

Note that $m$ is well-defined because $s$ and $h$ are continuous and, for $v \in\left[v_{m}, \hat{v}\right], h(v)$ is bounded away from zero and infinity because of the assumption $\hat{v}<\bar{v}$. Moreover, since $U(s(v) \mid v)=U^{*}(v)$ is strictly increasing in $v$ and $s(\widetilde{v})=\widetilde{v}$, it follows that $s(v)<v$ for $v \in\left[v_{m}, \hat{v}\right]$. Therefore we see that $m>0$. Because $f(\underline{v})>0$ and $f$ is continuous on $[\underline{v}, \bar{v})$, there exists $v_{0} \in\left(\underline{v}, v_{m}\right]$ and $\underline{f}>0$ such that $f(v) \geq \underline{f}$ for all $v \in\left[\underline{v}, v_{0}\right]$. The bounds $m$ and $\underline{f}$ are used next.

On the one hand, since $G$ is bounded, for $v \in\left[\widetilde{v}, v_{0}\right]$ we have

$$
G(v)-G\left(v_{m}\right)=G(v)-v_{m} \leq \hat{v} .
$$

On the other hand, for $v \in\left(\widetilde{v}, v_{0}\right]$ we have

$$
\begin{aligned}
G(v)-G\left(v_{m}\right) & =\int_{v_{m}}^{v} G^{\prime}(y) d y & & \\
& =\int_{v}^{v_{m}} \frac{h(y)}{y-s(y)} \frac{G(y)-s(G(y))}{h(G(y))} & & (\text { by }(20)) \\
& \geq m \int_{v}^{v_{m}} \frac{h(y)}{y-s(y)} d y & & \text { (definition of } m) \\
& \geq m \int_{v}^{v_{m}} \frac{f(y)}{y-s(y)} d y & & \\
& \geq m \underline{f} \int_{v}^{v_{0}} \frac{1}{y-s(y)} d y & & \left(v_{0} \leq v_{m} ; \text { definition of } \underline{f}\right)
\end{aligned}
$$




$$
\begin{aligned}
& \geq m \underline{f} \int_{v}^{v_{0}} \frac{1}{y-\widetilde{v}} d y \\
& =m \underline{f} \log \left(\frac{v_{0}-\widetilde{v}}{v-\widetilde{v}}\right) \rightarrow \infty \quad \text { as } v \downarrow \widetilde{v},
\end{aligned}
$$

showing (21) is violated for $v$ close to $\widetilde{v}$. This contradiction implies $(s, s)$ cannot be a regular equilibrium.

Proof of Lemma 2. Consider the equation for a linear inverse hazard rate

$$
\frac{1-F(v)}{f(v)}=\gamma v+\delta
$$

which can be restated as

$$
\frac{-f(v)}{1-F(v)}=-\frac{1}{\gamma v+\delta}
$$

If $\gamma=0$, then $\delta>0$ and integration of (22) yields

$$
\log (1-F(v))=-\frac{1}{\delta} v+\lambda,
$$

where $\lambda$ is a constant of integration. The condition $F(\underline{v})=0$ implies $\lambda=\underline{v} / \delta$, so

$$
F(v)=1-e^{-r(v-\underline{v})},
$$

where $r=1 / \delta>0$; and the condition $\lim _{v \rightarrow \bar{v}} F(v)=1$ implies $\bar{v}=+\infty$.

If $\gamma \neq 0$, integration of (22), along with the boundary conditions $F(\underline{v})=0$ and $F(\bar{v})=1$, yields

$$
F(v)=1-\left(\frac{\bar{v}-v}{\bar{v}-\underline{v}}\right)^{r}
$$

for some $r \neq 0$. Since $F$ must be increasing, we have $r>0$.

Proof of Proposition 3. The proof applies Proposition 1. If $s(v)=\alpha v+\beta$ on $[\widetilde{v}, \hat{v}]$, then $G(v)=(t-2 \beta) / \alpha-v$ so equation (2) is satisfied. If $F$ is given in (6), then (as in (5)) equation (1) becomes

$$
\frac{1}{r}=\frac{1-\alpha}{\alpha}(\hat{v}+\widetilde{v}-v)-\frac{\beta}{\alpha} \quad \forall v \in(\widetilde{v}, \hat{v}),
$$

which is satisfied if and only if

$$
\alpha=1 \text { and } \beta=-\frac{1}{r} \text {. }
$$

Therefore, on $[\widetilde{v}, \hat{v}]$ a regular piecewise-linear equilibrium strategy must be $s(v)=\alpha v+\beta=v-\frac{1}{r}$; conse- 
quently, the necessary condition $s(\widetilde{v})=\widetilde{v}$ if $\widetilde{v}>\underline{v}$ can never be satisfied, implying by (4) that $\widetilde{v}=\underline{v}$. Because contributions must be non-negative, we have

$$
\underline{v}-\frac{1}{r}=\widetilde{v}-\frac{1}{r} \geq 0
$$

Finally, the condition $s(\widetilde{v})+s(\hat{v})=t$ implicitly defines $\hat{v}$ as

$$
\hat{v}=t-\underline{v}+\frac{2}{r}
$$

The requirement that $\hat{v} \geq \underline{v}$ yields

$$
t \geq 2\left(\underline{v}-\frac{1}{r}\right)
$$

Conditions (25) and (26) are the necessary conditions stated in the proposition. If these conditions are satisfied, then $s$ given in (8) satisfies $a-d$ and (1)-(3) of Proposition 1; and with $\widetilde{v}=\underline{v}$, it follows that $(s, s)$ is a symmetric equilibrium.

Proof of Proposition 4. The proof applies Proposition 1. If $s(v)=\alpha v+\beta$ on $[\widetilde{v}, \hat{v}]$, then $G(v)=(t-2 \beta) / \alpha-v$ so equation (2) is satisfied. If $F$ is given by (7), then (as in (5)) equation (1) becomes

$$
\frac{1}{r}(\bar{v}-v)=(\hat{v}+\widetilde{v}-v) \frac{1-\alpha}{\alpha}-\frac{\beta}{\alpha} \quad \forall v \in(\widetilde{v}, \hat{v}),
$$

which is satisfied if and only if

$$
\alpha=\frac{r}{1+r} \text { and } \beta=-\frac{\bar{v}-(\hat{v}+\widetilde{v})}{1+r} .
$$

Therefore, on $[\widetilde{v}, \hat{v}]$ a regular piecewise-linear equilibrium strategy must be

$$
s(v)=\frac{r}{1+r} v-\frac{\bar{v}-(\hat{v}+\widetilde{v})}{1+r} .
$$

By the definition of $G$, we must have

$$
s(\widetilde{v})+s(\hat{v})=t
$$

or, using (29),

$$
t=\left(\frac{2+r}{1+r}\right)(\widetilde{v}+\hat{v})-\frac{2 \bar{v}}{1+r},
$$


which can be rewritten as

$$
\begin{aligned}
\widetilde{v}+\hat{v} & =\left(\frac{1+r}{2+r}\right) t+\frac{2 \bar{v}}{2+r} \\
& >\frac{(2+r) \underline{v}+r \bar{v}}{2+r}+\frac{2 \bar{v}}{2+r} \\
& =\bar{v}+\underline{v}
\end{aligned}
$$

and, since $\hat{v} \leq \bar{v}$ we must have $\widetilde{v}>\underline{v}$. When $\widetilde{v}>\underline{v}$, the necessary condition $s(\widetilde{v})=\widetilde{v}$ applies, and using (29) we obtain $\hat{v}=\bar{v}$. Therefore, $\widetilde{v}$ is implicitly determined by $s(\widetilde{v})+s(\bar{v})=t$, yielding

$$
\widetilde{v}=\frac{t(1+r)-r \bar{v}}{2+r}
$$

In order for the last expression to belong to $(\underline{v}, \bar{v})$ it must be that

$$
\frac{r \bar{v}+\underline{v}(2+r)}{1+r}<t<2 \bar{v}
$$

thus completing the proof of necessity of the Proposition.

The foregoing shows that if there exists a symmetric piecewise-linear regular equilibrium $(s, s)$, then on $[\widetilde{v}, \hat{v}]$ strategy $s$ has the form in (29). Note that the strategy $s$ in (29) satisfies conditions $a-d$. To show further that it constitutes an equilibrium we consider the case $\widetilde{v}>\underline{v}$, which is possible because $t \geq \frac{r \bar{v}+\underline{v}(2+r)}{1+r}$. Using (29), the necessary condition that $s(\widetilde{v})=\widetilde{v}$ now implies $\hat{v}=\bar{v}$, so the candidate equilibrium strategy is given by (10). This strategy has properties $a-d$ of Proposition 1 and satisfies the sufficient conditions (1)-(3). To complete the proof, it only remains to show that (4) is also satisfied. Because $t=s(\bar{v})+s(\widetilde{v})=s(\bar{v})+\widetilde{v}$, the remaining condition in (4) can be rewritten as

$$
(\bar{v}-(t-\widetilde{v}))(1-F(\widetilde{v})) \geq \bar{v}-t,
$$

which, after substituting for $F$ and rearranging terms, becomes

$$
\frac{\bar{v}-(t-\widetilde{v})}{\bar{v}-t}\left(\frac{\bar{v}-\widetilde{v}}{\bar{v}-\underline{v}}\right)^{r} \geq 1
$$

It now suffices to show (31) is satisfied. From the condition $t=\widetilde{v}+s(\bar{v})$, we obtain

$$
\widetilde{v}=\frac{t(1+r)-r \bar{v}}{2+r}
$$


Consequently, we have

$$
\bar{v}-(t-\widetilde{v})=\frac{(2+r)(\bar{v}-t)+t(1+r)-r \bar{v}}{2+r}=\frac{2 \bar{v}-t}{2+r}
$$

and

$$
\bar{v}-\widetilde{v}=\frac{(2+r) \bar{v}-t(1+r)+r \bar{v}}{2+r}=\frac{2 \bar{v}-t}{2+r}(1+r)
$$

so, to show that (31) is satisfied when $\widetilde{v}>\underline{v}$, we need to show

$$
\frac{(2 \bar{v}-t)^{r+1}}{(\bar{v}-t)} \frac{1}{2+r}\left(\frac{1}{\bar{v}-\underline{v}} \times \frac{1+r}{2+r}\right)^{r} \geq 1 \quad \forall t>\frac{(2+r) \underline{v}+r \bar{v}}{1+r} .
$$

Consider the function

$$
\frac{(2 \bar{v}-t)^{r+1}}{\bar{v}-t}
$$

on the left-hand side of (34). Its derivative with respect to $t$ is

$$
\begin{aligned}
\frac{-(r+1)(2 \bar{v}-t)^{r}(\bar{v}-t)+(2 \bar{v}-t)^{r+1}}{(\bar{v}-t)^{2}} & =\frac{(2 \bar{v}-t)^{r}}{(\bar{v}-t)^{2}}(-(r+1)(\bar{v}-t)+2 \bar{v}-t) \\
& =\frac{(2 \bar{v}-t)^{r}}{(\bar{v}-t)^{2}}(r t+(1-r) \bar{v}) \\
& \gtreqless 0 \Longleftrightarrow t \gtreqless \frac{r-1}{r} \bar{v} .
\end{aligned}
$$

Because

$$
\frac{(2+r) \underline{v}+r \bar{v}}{1+r}>\frac{r}{1+r} \bar{v}>\frac{r-1}{r} \bar{v}
$$

it follows that (35) is strictly increasing in $t$ for $t>\frac{(2+r) \underline{v}+r \bar{v}}{1+r}$. Therefore, for any $t>\frac{(2+r) \underline{v}+r \bar{v}}{1+r}$ we have

$$
\begin{aligned}
\frac{(2 \bar{v}-t)^{r+1}}{\bar{v}-t} & >\left.\frac{(2 \bar{v}-t)^{r+1}}{\bar{v}-t}\right|_{t=\frac{(2+r) \underline{v}+r \bar{v}}{1+r}} \\
& =(2+r)\left(\frac{\bar{v}-\underline{v}}{\bar{v}-(2+r) \underline{v}}\right)\left(\frac{2+r}{1+r}\right)^{r}(\bar{v}-\underline{v})^{r} .
\end{aligned}
$$

Using (36) we have

$$
\text { lhs of }(34) \geq\left[(2+r)\left(\frac{\bar{v}-\underline{v}}{\bar{v}-(2+r) \underline{v}}\right)\left(\frac{2+r}{1+r}\right)^{r}(\bar{v}-\underline{v})^{r}\right] \frac{1}{(2+r)}\left(\frac{1}{\bar{v}-\underline{v}} \times \frac{1+r}{2+r}\right)^{r}
$$




$$
\begin{aligned}
& =\frac{\bar{v}-\underline{v}}{\bar{v}-(2+r) \underline{v}} \\
& \geq \frac{\bar{v}-\underline{v}}{\bar{v}-\underline{v}}=1,
\end{aligned}
$$

establishing (34), thereby completing the proof.

Proof of Proposition 5. We proceed exactly as in the proof of Proposition 4 to derive that $\widetilde{v}>\underline{v}$ implies

$$
t>\frac{r \bar{v}+\underline{v}(2+r)}{1+r}
$$

contradicting the definition of the low-threshold case. Therefore, in the low-threshold case, regular piecewiselinear symmetric equilibria must have $\widetilde{v}=\underline{v}$. In this case $\hat{v}$ is implicitly determined by $s(\underline{v})+s(\hat{v})=t$, which, using (29), yields after rearrangement

$$
\hat{v}=\frac{t(1+r)+2 \bar{v}}{2+r}-\underline{v} .
$$

Substituting this value into (29) yields

$$
s(\underline{v})=\frac{r}{1+r} \underline{v}-\frac{\bar{v} r-t(1+r)}{(2+r)(1+r)}
$$

but the necessary condition $s(\underline{v}) \geq 0$ thus implies

$$
t \geq \frac{r}{1+r}(\bar{v}-\underline{v}(2+r))
$$

At the same time, we must have

$$
\underline{v} \leq \hat{v}=\frac{t(1+r)+2 \bar{v}}{2+r}-\underline{v}
$$

thus implying

$$
t \geq-\frac{2}{(1+r)}(\bar{v}-\underline{v}(2+r))
$$

and completing the proof of necessity of the Proposition.

The foregoing shows as well that the strategy (12) has properties $a-d$ of Proposition 1 and satisfies the sufficient conditions (1)-(3). Since $\widetilde{v}=\underline{v}$, condition (4) is automatically satisfied. This establishes the sufficiency of Proposition 5.

Proof of Proposition 8. Because

$$
v-\rho \frac{1-F(v)}{f(v)}=\left(\frac{1-\rho+r}{r}\right) v-(1-\rho) \frac{\bar{v}}{r}
$$


Lemma 3 shows the optimal mechanism provides the good if and only if

$$
c \leq\left(\frac{(1-\rho)+r}{r}\right)\left(v_{1}+v_{2}\right)-\frac{2(1-\rho) \bar{v}}{r}
$$

which is equivalent to

$$
v_{1}+v_{2} \geq \frac{2(1-\rho) \bar{v}+c r}{(1-\rho)+r} .
$$

Note how

$$
t=\frac{c r(2+r)+2 \bar{v}(1-\rho(1+r))}{(1+r)((1-\rho)+r}
$$

and $c r \geq \underline{v}((1-\rho)+r)+(r-(1-\rho)) \bar{v}$ imply

$$
t \geq \frac{\underline{v}(2+r)+\bar{v} r}{(1+r)}
$$

the definition of high-threshold case in (9). Therefore, the strategy in (10) is part of a symmetric equilibrium where completion happens if and only if $s\left(v_{1}\right)+s\left(v_{2}\right) \geq t$, or

$$
\begin{aligned}
v_{1}+v_{2} & \geq \frac{2 \bar{v}+t(1+r)}{2+r} \\
& =\frac{2(1-\rho) \bar{v}+c r}{(1-\rho)+r},
\end{aligned}
$$

using (10) and the choice of $t$ in the Proposition, showing the provision region in this subscription-game equilibrium coincides with that in the optimal mechanism (see (37)). To conclude, note that in (10) we have $\widetilde{v}>\underline{v}$, so that $U_{i}^{*}(\underline{v})=0$. Therefore, $\varphi=0$ is optimal.

Proof of Proposition 9. Note how the choice of $t$ in the Proposition and $c r \leq \underline{v}((1-\rho)+r)+(r-(1-\rho)) \bar{v}$ imply

$$
t \leq \frac{\underline{v}(2+r)+\bar{v} r}{(1+r)}
$$

the definition of low-threshold case in (11). Therefore, if the strategy in (12) is part of a symmetric equilibrium, proceeding as for the proof of Proposition 8, one may show that the completion region induced by (12) is the same specified in Lemma 3. The choice of the entry fee $\varphi$ delivers

$$
0=U_{i}^{*}(\underline{v})=(\underline{v}-s(\underline{v}))(1-F(\hat{v}))-\varphi
$$

using (12). Therefore, we just need to verify the additional conditions specified in Proposition 5. When 
$\bar{v}-\underline{v}(2+r)>0$ we need

$$
t \geq \frac{r}{1+r}(\bar{v}-\underline{v}(2+r))
$$

which, after substitution, reduces to

$$
c r>\bar{v}(r+\rho-1)-\underline{v} r(1+r-\rho),
$$

and this condition is required by the statement of this Proposition. When $\bar{v}-\underline{v}(2+r)<0$ we need

$$
t \geq-\frac{2}{1+r}(\bar{v}-\underline{v}(2+r))
$$

which, after substitution, yields

$$
c r>2 \underline{v}(1+r-\rho)-2(1-\rho) \bar{v},
$$

and this condition is equivalent to the assumption that the collector does not produce the good with probability one in the optimal mechanism: $\operatorname{cr}>2((1-\rho)+r) \underline{v}-2(1-\rho) \bar{v}$.

Proof of Proposition 10. The proof proceeds with the same steps of the proof of Proposition 7, so $G(v)=$ $k_{1}-v$ for some constant $k_{1}$. Equation (1) now reduces to

$$
s^{\prime}(v)=\frac{r(v-s(v))}{\bar{v}-k_{1}+v}
$$

a nonconstant solution to this differential equation must have the form

$$
s(v)=\frac{k_{1}-\bar{v}+r v}{1+r}+k_{2}\left(k_{1}-\bar{v}-v\right)^{-r}
$$

for some constant $k_{2}$. With the same steps as in the proof of Proposition 7, one can establish that $s$ cannot be an equilibrium when $k_{2} \neq 0$, while the conditions in this Proposition imply that $s$ cannot be an equilibrium when $k_{2}=0$, because $s(v)$ is not a valid linear equilibrium.

Proof of Proposition 11. Consider a symmetric equilibrium of the contribution game. Exactly as in Proposition 1, define the boundary that separates the region of the type space where completion occurs from the no-completion region as $(v, G(v))$. From Proposition 1 in Barbieri and Malueg, (forthcoming (a)) we have

$$
G^{\prime}(v)=-\frac{f(v)}{v} \frac{G(v)}{f(G(v))}
$$


If the equilibrium maximizes the sellers utility when the cost of the public good is $c_{m}$, we have from Lemma 3 that $w(v)+w(G(v))=c_{m}$; hence, after differentiation and substitution in the previous equation, we obtain

$$
\kappa(v) \equiv \frac{w^{\prime}(v) v}{f(v)}=\frac{w^{\prime}(G(v)) G(v)}{f(G(v))}=\kappa(G(v)) .
$$

Consider now the type $v_{m}$ that solves $w\left(v_{m}\right)+w\left(v_{m}\right)=c_{m}$. By definition of $G$ we must have $G\left(v_{m}\right)=v_{m}$. Moreover, for all types smaller than $v_{m}$, the corresponding $G\left(v_{m}\right)$ will be larger than $v_{m}$. Consequently, $\kappa^{\prime}\left(v_{m}\right)=0$ : If $\kappa$ did not have either a maximum or a minimum at $v_{m}$, it would be impossible to find two types, one on each side of $v_{m}$, such that $\kappa$ assumes the same value, for a sufficiently small neighborhood of $v_{m}$. Repeating the same reasoning for all values of the cost in $\left(c_{m}-\epsilon, c_{m}+\epsilon\right)$ we obtain that $\kappa^{\prime}=0$ on an interval around $v_{m}$. Equation (15) is a simple rearrangement of the condition $\kappa^{\prime}=0$, using the definition of $\kappa$ above.

Proof of Proposition 12. By contradiction, assume such $F$ exists. Note that $F$ satisfies (15), the right-hand side of which does not depend on $\rho$. The left-hand side of (15) depends on $\rho$ through the definition of $w$ in Lemma 3:

$$
w(v)=v-(1-\rho) \frac{1-F(v)}{f(v)},
$$

which delivers

$$
w^{\prime}(v)=1+(1-\rho)\left[\frac{f^{\prime}(v)}{f(v)} \times \frac{1-F(v)}{f(v)}+1\right] .
$$

Therefore, indicating by $z(v) \equiv \frac{f^{\prime}(v)}{f(v)} \times \frac{1-F(v)}{f(v)}+1$ the term in square brackets, which is independent of $\rho$ by the contradiction hypothesis, the left-hand side of $(15)$ is

$$
\frac{(1-\rho) z^{\prime}(v)}{1+(1-\rho) z(v)}
$$

Fix now a $v$ for which (15) must hold for a value of $\rho$ denoted $\rho_{1}$. Consider a different value $\rho_{2}$ that is sufficiently close to $\rho_{1}$ so that $v$ is inside that open set of players' valuations for which (15) holds for $\rho=\rho_{2}$ as well. For both values of $\rho$, equation (15) holds. Therefore, we obtain

$$
\frac{\left(1-\rho_{1}\right) z^{\prime}(v)}{1+\left(1-\rho_{1}\right) z(v)}=\frac{\left(1-\rho_{2}\right) z^{\prime}(v)}{1+\left(1-\rho_{2}\right) z(v)}
$$

implying $z^{\prime}(v)=0$. Since we fixed an arbitrary $v$, the condition $z^{\prime}(v)=0$ must hold not just for a specific 
valuation, but for an open interval of $v$. Therefore, equation (15) simplifies to

$$
\frac{f^{\prime}(v)}{f(v)}=\frac{1}{v}
$$

which, upon integration, yields $f(v)=k_{1} v$, for some $k_{1}>0$. Further integration yields $F(v)=\frac{k_{1}}{2} v^{2}+k_{2}$, where $k_{2}$ is another constant of integration. Note that $k_{2}$ must be strictly smaller than 1 , for $F$ to represent a meaningful probability distribution. Substituting these values into the definition of $z$ we obtain

$$
z(v)=\frac{1}{2}+\frac{1-k_{2}}{k_{1} v^{2}}
$$

therefore,

$$
z^{\prime}(v)=-\frac{2\left(1-k_{2}\right)}{k_{1} v^{3}}
$$

which satisfies the differential equation $z^{\prime}(v)=0$ only if $k_{2}=1$. As noted above, this value is not admissible if $F$ is to represent a probability distribution, and we obtain a contradiction.

\section{References}

Andreoni, J., (1998), Toward a theory of charitable fund-raising, Journal of Political Economy 106, 11861203.

Admati, Anat R., and Motty Perry (1991), Joint projects without commitment, Review of Economic Studies $58,259-276$.

Alboth, Dirk, Anat Lerner, and Jonathan Shalev (2001), Profit maximizing in auctions of public goods, Journal of Public Economic Theory 3, 501-525.

Bagnoli, Mark, and Barton L. Lipman (1989), Provision of public goods: fully implementing the core through private contributions, Review of Economic Studies 56: 583-601.

Barbieri, Stefano, and David A. Malueg, Private provision of a discrete public good: efficient equilibria in the private-information contribution game, forthcoming (a) in Economic Theory.

Barbieri, Stefano, and David A. Malueg, Private provision of a discrete public good: continuous-strategy equilibria in the private-information subscription game, forthcoming (b) in Journal of Public Economic Theory.

Cornelli, F., (1996), Optimal selling procedures with fixed costs, Journal of Economic Theory 71, 1-30.

Holmström, Bengt, and Roger B. Myerson (1983), Efficient and durable decision rules with incomplete information, Econometrica 51: 1799-1820.

Laussel, Didier, and Thomas R. Palfrey (2003), Efficient equilibria in the voluntary contributions mechanism with private information, Journal of Public Economic Theory 5, 449-478.

Ledyard, John O., and Thomas R. Palfrey (1999), A characterization of interim efficiency with public goods, Econometrica 67, 435-448.

Marx, Leslie M., and Steven A. Matthews (2000), Dynamic voluntary contribution to a public project, Review of Economic Studies 67: 327-358. 
Martimort, David, and Humberto Moreira (2007), Common agency with informed principals, mimeo.

Menezes, Flavio M., Paulo K. Monteiro, and Akram Temimi (2001), Private provision of discrete public goods with incomplete information, Journal of Mathematical Economics 35, 493-514.

Myerson, R., (1981), Optimal auction design, Mathematics of Operations Research 6, 58-73.

Myerson, Roger B., and Mark Satterthwaite (1983), Efficient mechanisms for bilateral trading, Journal of Economic Theory 29, 265-281.

Palfrey, Thomas R., and Howard Rosenthal (1984), Participation and the provision of discrete public goods: a strategic analysis, Journal of Public Economics 24, 171-193. 